\title{
Investigating signal properties of UHE particles using in-ice radar for the RET experiment
}

\section{Dieder Van den Broeck, ${ }^{*}$ Enrique Huesca Santiago, " Uzair Latif* and Vesna Lukic* on behalf of the Radar Echo Telescope Collaboration}

(a complete list of authors can be found at the end of the proceedings)

\author{
${ }^{a}$ Vrije Universiteit Brussel, \\ Boulevard de la Plaine 2, 1050 Ixelles, Brussel \\ E-mail: dieder.jan.van.den.broeck@vub.be, enrique.huesca.santiago@vub.be, \\ uzair.abdul.latif@vub.be, vesna.lukic@vub.be
}

The Radar Echo Telescope (RET) experiment plans to use the radar technique to detect Ultra-High Energy (UHE) cosmic rays and neutrinos in the polar ice sheets. Whenever an UHE particle collides with an ice molecule, it produces a shower of relativistic particles, which leaves behind an ionization trail. Radio waves can be reflected off this trail and be detected in antennas. It is critical to understand such a radar signal's key properties as that will allow us to do vertex, angular and energy reconstruction of the primary UHE particle. We will discuss various simulation methods, which will fundamentally rely on ray tracing, to recreate the radar signal and test our reconstruction methods.

$37^{\text {th }}$ International Cosmic Ray Conference (ICRC 2021)

July 12th - 23rd, 2021

Online - Berlin, Germany

\footnotetext{
${ }^{*}$ Presenter
} 


\section{Introduction}

In this proceeding we discuss the signal properties of the in-ice radar echo signal via two models and discuss their use for reconstruction: RadioScatter [1], a detailed microscopic scattering code ${ }^{1}$ that works at the individual particle level (using GEANT4 or other Monte Carlo cascade generators); and a macroscopic approach, MacroScatter, based on the plasma's macroscopic reflection profile (currently under development at the time of this writing). These independent models compute the return signal of the radar echo and, by investigating the signal properties (power, duration, frequency shift, etc.), we expect to perform vertex, angular and energy reconstruction of the incoming particle. For consistency, these results are cross-checked with a basic line model derived from first principles. To finish, we show an example of vertex reconstruction using a geometric optics (ray tracing) approach in a polar-like ice profile.

\section{The Cascade}

An UHE cascade within a dense medium will leave an ionized plasma in its wake. This plasma, if dense enough, will reflect incident radio waves to a receiver [2-4]. For a cascade induced in ice at our energy range $(>10 \mathrm{PeV})$, this will result in a compact, highly elongated shower with a very dense core (Fig 1), since the majority of high energy cascade particles remain close to the axis of development.

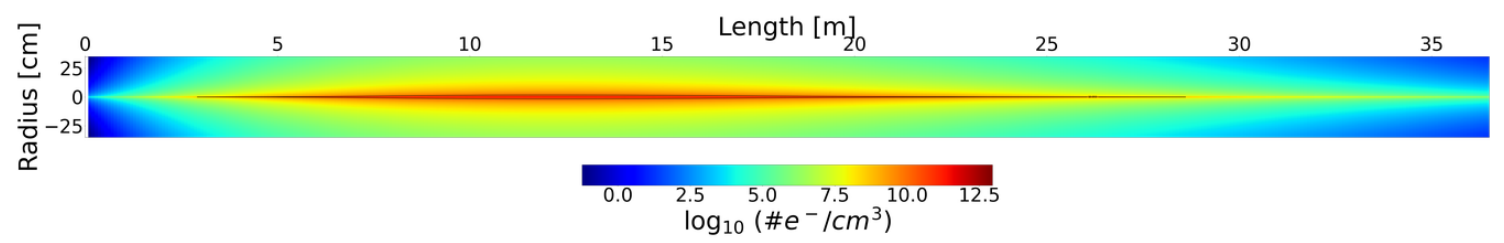

Figure 1: Plasma's density distribution for a $10 \mathrm{PeV}$ cascade, used by MacroScatter following the parametrization from [4].

Assuming elastic interactions during the plasma lifetime, the amount of scattered power equals the power which is taken from the incoming wave. The power transmitted through the plasma is obtained using

$$
\frac{P(l)}{P_{0}}=\frac{\Phi(l)}{\Phi_{0}}=e^{-\int_{0}^{l} \beta(l) d l}=e^{-\int_{0}^{l} \frac{d l}{\delta(l)}},
$$

where $l$ is the distance along the wave-vector $\vec{k}$ of the radio wave, $\beta=\operatorname{Im}(\vec{k})$ is the attenuation due to propagation through the plasma (not to be confused with attenuation by the medium, which is treated separately) and $\delta=\frac{1}{\beta}$ is the skin depth. Both RadioScatter and MacroScatter use these parameters to capture relevant physics like the collisional damping of the free electrons $\beta=\beta\left(\omega_{T X}, \omega_{\text {plasma }}, \omega_{\text {collisions }}\right)$. Both parameters are shown below in Fig 2.

Since the skin depth is larger than the dimensions of the plasma, even at its peak value, $\delta \sim O\left(10^{4} \mathrm{~m}\right)$, the plasma is a mostly transparent object. Nevertheless, detailed simulations [5] and experimental observations $[6,7]$ show that the amount of reflected power is enough to detect for primary neutrino energies in the $10-100 \mathrm{PeV}$ range and beyond.

\footnotetext{
${ }^{1}$ https://github.com/prchyr/RadioScatter
} 

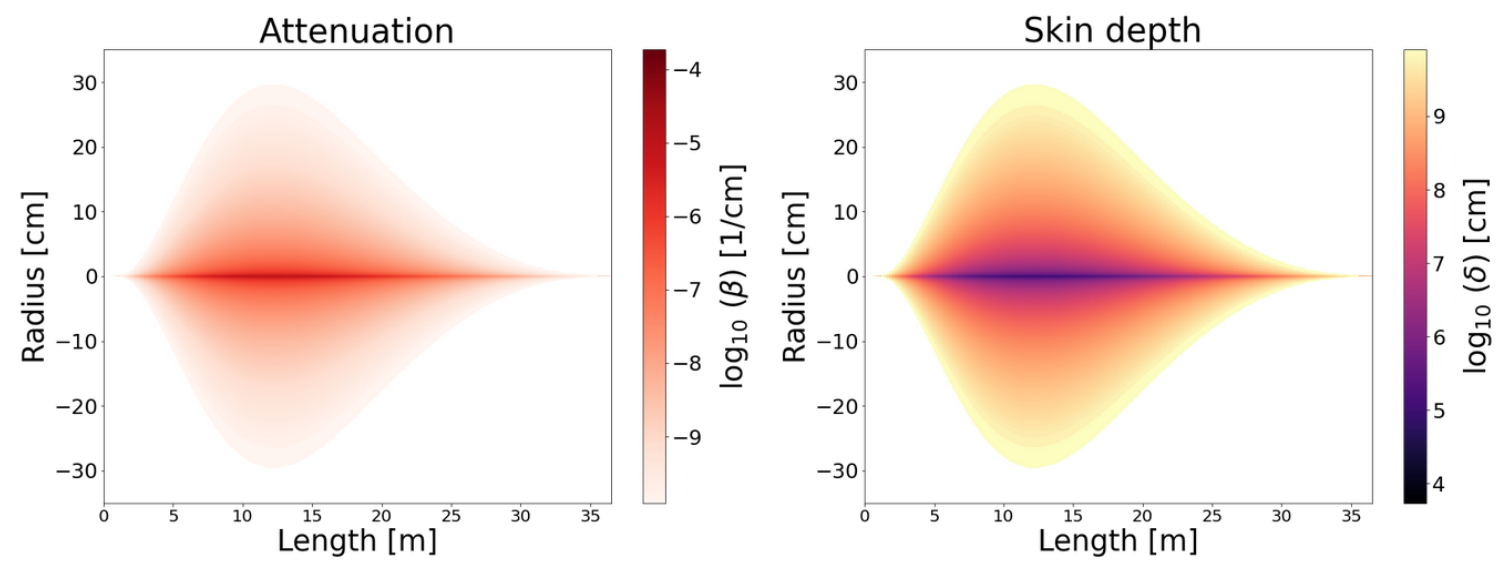

Figure 2: The equivalent plots of the attenuation coefficient $\beta$ and the skin-depth $\delta$ for the shower in Fig. 1. MacroScatter relies on the fact that most of the scatter is expected from the inner region of the cascade.

\section{Energy Scaling and Directional Stretching}

The computational time required to simulate a particle cascade is proportional to the primary energy, therefore lower energy primaries are scaled in energy and stretched geometrically to simulate higher energy cascades. In this scaling/stretching procedure, a shower with e.g. a $10 \mathrm{GeV}$ primary is scaled up in energy, and the shower is stretched along the propagation axis to reach a specific target primary energy. We find good agreement in the profiles of scaled and stretched cascades compared to GEANT simulations and cascade parameterizations using the following stretching relation,

$$
\begin{gathered}
x_{\text {updated }}=x s_{x}+168.3 \log \left(E_{t} / E_{p}\right) \mathrm{mm} \\
s_{x}=s_{v x}-\left(s_{v x}-1\right) e^{\left(-\left(0.6 x_{\text {max }} / x\right)^{0.6}\right)} \\
s_{v x}=\log _{10}\left(E_{t} / 78.6\right) / \log _{10}\left(E_{p} / 78.6\right) \\
x_{\text {max }}=992.5 \log _{10}\left(0.00336 E_{p}\right) \mathrm{mm},
\end{gathered}
$$

where $x_{\text {updated }}$ refers to the nonlinear stretching applied along the $x$-axis, $x$ refers to the distance along the axis of the cascade propagation, $E_{t}$ and $E_{p}$ are the target and primary energies respectively. The Heitler formula [8] is used to derive the stretch factor $s_{v x}$, from a primary energy to a target energy.

\section{Towards Angular Reconstruction}

\subsection{Signal Properties}

The properties of the radar signal can be investigated to reconstruct the properties of the primary particle. Previous work has looked at the relationship between the signal duration and the cascade azimuth angle $\phi$ (the angle subtended by the TX-RX baseline and the cascade's momentum direction vector) [9], using a simulation of a bi-static radar scatter off a line of randomly spaced point sources (perfect reflectors) which emit spherically. In this section, RadioScatter and MacroScatter will be compared under a similar simple geometry $(\mathrm{TX}=[0,0,0], \mathrm{RX}=[500,0,0]$, vertex $=[250,-250,0]$ with 
a random angular distribution in the $\mathrm{x}$-y plane) keeping the line model as a reference. All models account for the cascade propagation, ionization lifetime, and retardation effects.

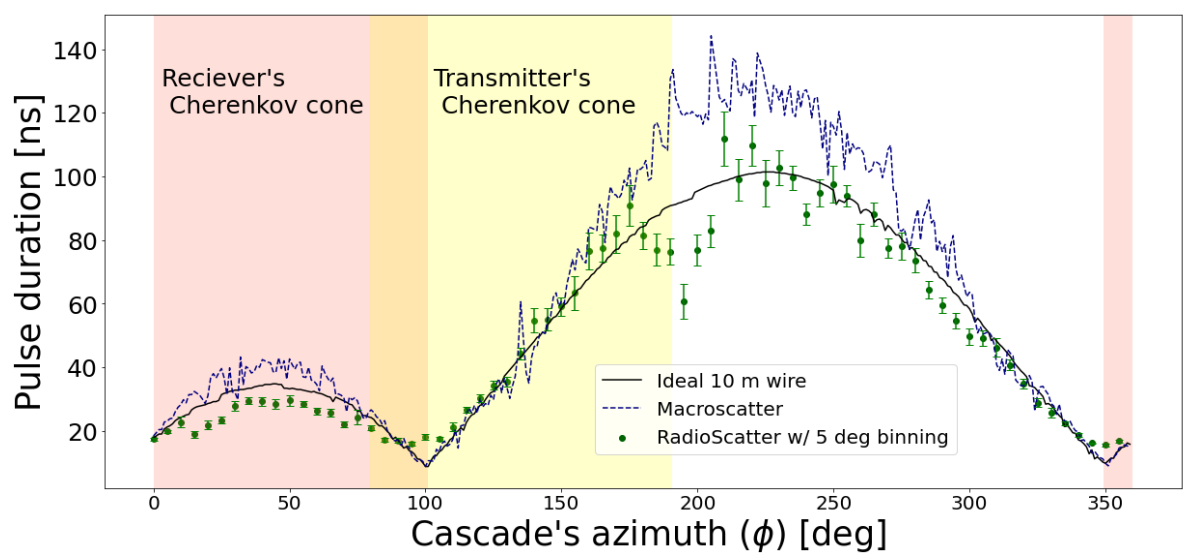

Figure 3: Receiver signal duration vs azimuth angle $\phi$. RadioScatter used 1000 trial events and 5 degree binning with error bars indicating the standard error. MacroScatter and the ideal line simulations were run for every 1 degree.

Fig 3 shows a comparison of the received pulse duration as a function of $\phi$ for all three simulation methods. Indicated in the figure are some regions of interest. The Cherenkov cone, defined by angles less than the Cherenkov angle (measured with respect to the cascade axis) is indicated with respect to both the receiver and transmitter. Though the radar echo method does not measure Askaryan (radio Cherenkov [10]) radiation, the geometry responsible for it also results in observable signal features of the radar echo signal. The general shape for all three simulations is similar, with a nadir at the edges of Cherenkov angle with respect to the receiver, and a maximum at the opposing angle. RadioScatter also shows structure at the transmitter's Cherenkov angle. Investigations are under way to explain the lack of a similar structure in the idealized and macroscopic simulations.

\section{Towards Energy Reconstruction}

Using RadioScatter, it is possible to simulate a large number of events with a range of primary energies, cascade positions and directions, and calculate properties of the return signal. Basic linear regression methods and Machine Learning (ML) techniques can be applied to these properties to predict the energies and calculate the resolution. As a first application of ML to the problem of energy resolution, a total of 5,000 events were simulated in the energy range of $10^{14}-10^{20} \mathrm{eV}$, using $60 \%$ for training and $40 \%$ for testing, with isotropic directions and positions within a total volume of $600 \times 600 \times 600 \mathrm{~m}^{3}$, using the same transmitter-receiver configuration outlined previously.

We trained a very simple neural network using only the integrated power and duration of the return signal as predictors. The architecture consisted of an input and output layer, and 1 hidden layer of 5 neurons. The 'ReLu' activation function was used in all layers except the output layer, where a linear activation was used. The batch size was 512. The mean squared error was minimised using the 'adadelta' optimiser. The network was trained for 200 epochs, using early stopping with a patience of 10 epochs. An example output of this procedure is given in Fig. 4. Quantitative results 


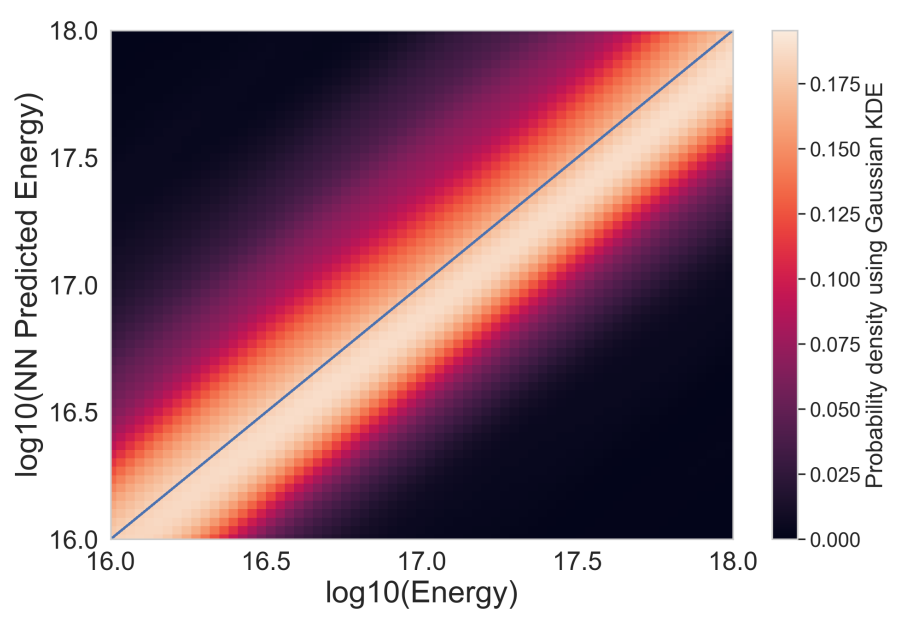

Figure 4: The estimated probability density of the Neural Network predictions vs the true Primary Energy values. The energies are plotted on a $\log _{10}$ scale. The Gaussian kernel density estimate was used to estimate the probability density, without normalisation. The blue line on the diagonal of the corresponds to the line $y=x$, where the energy predictions would agree perfectly with the true primary energies.

of this study and an expanded ML implementation incorporating other predictor variables will be presented in a forthcoming article.

\section{Towards Vertex Reconstruction - Ice Raytracing}

Ice raytracing is simply the exercise of tracing radio wave rays as they propagate through ice [11-13]. Rays are refracted in the ice sheet owing to the depth-dependent density, and therefore the depth-dependent index of refraction. Neglecting small-scale density fluctuations, the top $\sim 200 \mathrm{~m}$ of polar ice sheets have an approximately exponential density profile (details can be found in Ref. [14]).

We note that raytracing is used for vertexing in this study because it is computationally efficient, but it does not capture all of the propagation effects of polar ice on radio [15-17].Small scale density fluctuations can produce frequency-dependent propagation effects, that must be modelled more accurately. However, such effects require finite-difference time-domain or parabolic equation modeling [18], which are computationally more expensive. Therefore, though it is an approximation, ray tracing is used here for vertexing with an understanding of its limitations.

Fig. 5 shows a voltage-time trace and spectrogram for a shower that was simulated with RadioScatter using raytracing. Two pulses are visible in Fig. 5: one with the shortest path length from the shower to the receiver and the other one which reflects off the air/ice interface on its way to the receiver. Both of these pulses are reflected from the shower due to the shower being illuminated by the transmitter CW. A chirp-like behavior can be seen in the spectrogram for both pulses. The chirp behavior can be exploited in trigger designs.

\subsection{Vertex Reconstruction}

In this simple example, vertexing is done using interferometry which requires the use of multiple receivers. Interferometry involves using the relative 'hit' times of pulses in the receiving antennas to calculate the direction of the incoming signal and the distance to the source. A minimization 

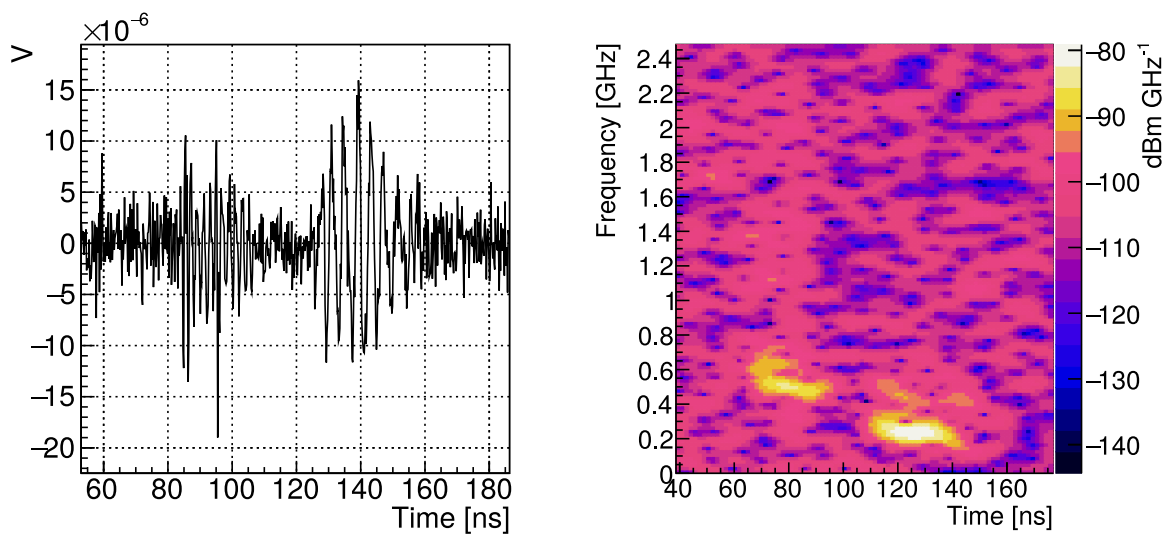

Figure 5: Voltage-time graph and spectrogram for an event simulated with RadioScatter with raytracing turned ON. In this case the shower is at $(0 \mathrm{~m}, 20 \mathrm{~m},-2 \mathrm{~m})$ and is propagating in the direction of the vector $(0,+1,-1)$. The transmitting antenna is at $(0 \mathrm{~m}, 10 \mathrm{~m},-15 \mathrm{~m})$ and is transmitting at a $\mathrm{CW}$ at a frequency of $0.5 \mathrm{GHz}$ with a power of $160 \mathrm{~W}$. The receiving antenna is at $(0 \mathrm{~m}, 20 \mathrm{~m},-20 \mathrm{~m})$. The plasma lifetime was set to be $10 \mathrm{~ns}$. The energy of the primary particle was set at $100 \mathrm{PeV}$. Noise is added.

procedure is performed where raytracing is used to simulate 'hit' times from varying sources until the relative 'hit' times match the ones extracted from data (or the RadioScatter simulation in this case).

In order to do vertex reconstruction, a dummy station configuration was used. There were three rings of receiving antennas in the dummy station, with each ring having nine equally spaced antennas, a radius of $200 \mathrm{~m}$ and depths of $-1480 \mathrm{~m},-1500 \mathrm{~m}$ and $-1520 \mathrm{~m}$ respectively. The rings were centered at the origin. The transmitter was placed at $(0 \mathrm{~m}, 0 \mathrm{~m},-500 \mathrm{~m})$ and the shower vertex was placed at $(0 \mathrm{~m}, 300 \mathrm{~m},-5 \mathrm{~m})$ and was propagating in the direction of the vector $(0,-0.2,-1)$. The relative hit times were obtained from the final waveforms for each receiver and were used to perform vertex reconstruction using the raytracing minimization procedure. The vertex's $\mathrm{X}, \mathrm{Y}$, and $\mathrm{Z}$ values were reconstructed within $\sim 5 \mathrm{~m}$ of the actual values.

This simple interferometric method is common to any in-ice radio experiment. RET can, however, perform vertex reconstruction in additional ways common to other radar systems, e.g., achieving ranging via transmitter modulation. This in conjunction with other signal observables (notably those seen to vary as a function of angle, as in Fig. 3) has the potential to provide vertexing in a single antenna, which will be explored in a forthcoming article.

\section{Conclusion}

We presented two different methods to compute the return signal from the reflection of a radar pulse off the ionization deposit left by a particle cascade. First, the geometrical setup and parameters of the simulation were shown. There was good agreement in the pulse duration vs. the azimuthal angle between RadioScatter and the simplified line model. The fact that the azimuthal angle and the pulse duration are correlated signifies the potential for angular reconstruction. Second, using the macroscopic approach, we showed the parameterized density distribution, the skin depth, and the effective scattering area of the cascade. We also explored how signal features obtained from 
RadioScatter can be used as inputs to low-level machine learning algorithms to estimate the energy of the incoming particle with the associated resolution. Finally, an example of vertex reconstruction using raytracing in a realistic ice profile was shown. In this procedure, the original geometrical setup was embedded in a larger configuration of antennas, as the raytracing vertexing method required multiple receivers. The vertex was reconstructed from the simulated RadioScatter signals within $\sim 5 \mathrm{~m}$ of the true position.

\section{Acknowledgements}

RET-CR is supported by the National Science Foundation under award numbers NSF/PHY2012980 and NSF/PHY-2012989. This work is also supported by the Flemish Foundation for Scientific Research FWO-12ZD920N, the European Research Council under the EU-ropean Unions Horizon 2020 research and innovation programme (grant agreement No 805486), and the Belgian Funds for Scientific Research (FRS-FNRS). A. Connolly acknowledges support from NSF Award \#1806923. S. Wissel was supported by NSF CAREER Awards \#1752922 and \#2033500. DZB is grateful for support from the U.S. National Science Foundation-EPSCoR (RII Track-2 FEC, award ID 2019597). We express our gratitude to R. Dallier, L. Martin, J-L. Beney and the CODALEMA experiment for providing electronics and hardware to be used in the surface radio stations of RET-CR. Computing resources were provided by the Ohio Supercomputer Center.

\section{References}

[1] S. Prohira and D. Besson, Particle-level model for radar based detection of high-energy neutrino cascades, Nucl. Instrum. Meth. A 922 (2019) 161 [1710.02883].

[2] P. Blackett and C. Lovell, Radio echoes and cosmic ray showers, Proc. Roy. Soc A 177 (1941) 183.

[3] P. Gorham, On the possibility of radar echo detection of ultrahigh-energy cosmic ray induced and neutrino induced extensive air showers, Astropart. Phys. 15 (2001) 177 [hep-ex/0001041].

[4] K.D. de Vries, K. Hanson, T. Meures and A. O'Murchadha, On the feasibility of RADAR detection of high-energy cosmic neutrinos, PoS ICRC2015 (2015) 1168 [1511.08796].

[5] S. Prohira et al., The Radar Echo Telescope for Cosmic Rays: Pathfinder Experiment for a Next-Generation Neutrino Observatory, 2104.00459.

[6] M. Chiba et al., Reflection of microwave from energy deposit by $x$-ray irradiation in rock salt, SUSY07 (2007) .

[7] S. Prohira et al., Suggestion of Coherent Radio Reflections from an Electron-Beam Induced Particle Cascade, Phys. Rev. D 100 (2019) 072003 [1810 . 09914].

[8] W. Heitler, Quantum theory of radiation (1954). 
[9] D.V.d. Broeck, Radar detection of high energy neutrino cascades. Study of signal features through first principle simulations, Ph.D. thesis, Vrije Universiteit Brussel, 2020.

[10] G.A. Askar'yan, Excess negative charge of an electron-photon shower and its coherent radio emission, Sov. Phys. JETP 14 (1962) 441.

[11] U.A. Latif, Towards measurement of UHECR with the ARA experiment, Ph.D. thesis, University of Kansas, 2020.

[12] C. Glaser et al., NuRadioMC: Simulating the radio emission of neutrinos from interaction to detector, Eur. Phys. J. C 80 (2020) 77 [1906. 01670].

[13] U.A. Latif, "Iceraytracing." https://github.com/uzairlatif90/IceRayTracing, 2019.

[14] J. Kelley, M.-Y. Lu, D. Seckel, Y. Pan, D.Z. Besson and F.T.A. Collaboration, Observation of two deep, distant $(1.4,4) \mathrm{km}$ impulsive RF transmitters by the Askaryan Radio Array (ARA)., PoS ICRC2017 (2018) 1030.

[15] S.W. Barwick et al., Observation of classically 'forbidden' electromagnetic wave propagation and implications for neutrino detection, JCAP 1807 (2018) 055 [1804 . 10430].

[16] P. Allison et al., Long-baseline horizontal radio-frequency transmission through polar ice, JCAP 12 (2020) 009 [1908 . 10689].

[17] C. Deaconu, A.G. Vieregg, S.A. Wissel, J. Bowen, S. Chipman, A. Gupta et al., Measurements and Modeling of Near-Surface Radio Propagation in Glacial Ice and Implications for Neutrino Experiments, Phys. Rev. D 98 (2018) 043010 [1805 . 12576].

[18] RADAR Echo Telescope collaboration, Modeling in-ice radio propagation with parabolic equation methods, Phys. Rev. D 103 (2021) 103007 [2011. 05997]. 


\section{Full Authors List: Radar Echo Telescope Collaboration}

S. Prohira ${ }^{1}$, K.D. de Vries ${ }^{2}$, P. Allison ${ }^{1}$, J. Beatty ${ }^{1}$, D. Besson ${ }^{3,4}$, A. Connolly ${ }^{1}$, P. Dasgupta ${ }^{5}$, C. Deaconu ${ }^{6}$, S. De Kockere ${ }^{2}$, D.

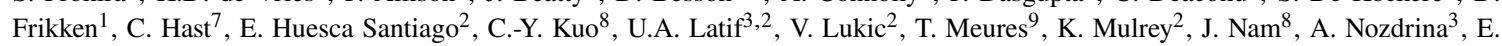
Oberla $^{6}$, J.P. Ralston ${ }^{3}$, C. Sbrocco ${ }^{1}$, R.S. Stanley ${ }^{2}$, J. Torres ${ }^{1}$, S. Toscano ${ }^{5}$, D. Van den Broeck ${ }^{2}$, N. van Eijndhoven ${ }^{2}$, and S. Wissel ${ }^{10,11}$

${ }^{1}$ Department of Physics, Center for Cosmology and AstroParticle Physics (CCAPP), The Ohio State University, Columbus OH, USA

${ }^{2}$ Vrije Universiteit Brussel, Brussel, Belgium

${ }^{3}$ University of Kansas, Lawrence, KS, USA

${ }^{4}$ National Research Nuclear University, Moscow Engineering Physics Institute, Moscow, Russia

${ }^{5}$ Université Libre de Bruxelles, Brussels, Belgium

${ }^{6}$ Enrico Fermi Institute, Kavli Institute for Cosmological Physics,Department of Physics, University of Chicago, Chicago, IL, USA

${ }^{7}$ SLAC National Accelerator Laboratory, Menlo Park, CA, USA

${ }^{8}$ National Taiwan University, Taipei, Taiwan

${ }^{9}$ University of Wisconsin-Madison, Madison, WI, USA

${ }^{10}$ Departments of Physics and Astronomy \& Astrophysics,Institute for Gravitation and the Cosmos, Pennsylvania State University, University Park, PA, USA

${ }^{11}$ California Polytechnic State University, San Luis Obispo CA, USA 